\title{
A Cross-Cultural STUdy of Purposive "TRAITS OF ACTION": MEASUREMENT INVARIANCE OF SCALES BASED ON THE ACTION-TRAIT THEORY OF HUMAN MOTIVATION USING EXPLORATORY STRUCTURAL EQUATION MODELING
}

\author{
LARRY C. BERNARD* \\ Loyola Marymount University \\ JAN CIECIUCH \\ Cardinal Stefan Wyszyński University \\ and University of Zurich
}

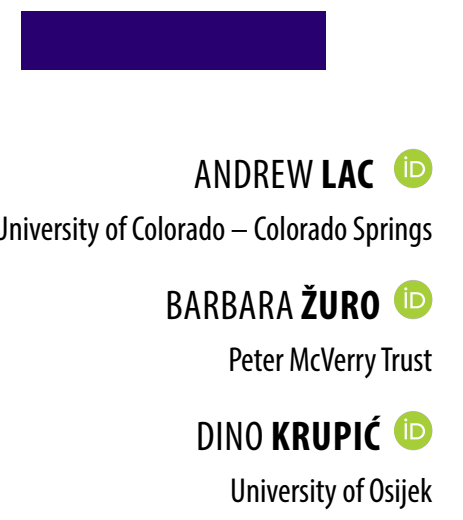

MICHAEL RICHTER

Liverpool John Moores University

NICOLAS SILVESTRINI

University of Geneva

BETTINA VON HELVERSEN

University of Basel

\begin{abstract}
The Action-Trait theory of human motivation posits that individual differences in predispositional traits of action may account for variance in contemporary purposeful human behavior. Prior research has supported the theory, psychometric properties of scales designed to assess the motive dimensions of the theory, and the utility of these scales to predict an array of behaviors, but this is the first study to evaluate the cross-linguistical invariance of the 15 -factor theoretical model. This study evaluated translations of the English language 60-item Quick AIM in 5 samples - Croatian $(N=614)$, French $(N=246)$, German $(N=154)$, Polish $(M=314)$, and U.S. English $(N=490)-$ recruited from 4 countries (Croatia, Poland, Switzerland, and the U.S.). Exploratory structural equation modeling (ESEM) supported the theoretical model on which the traits of action are based and scrutinized the measurement invariance (configural, metric, scalar invariance) of the scale across the languages.
\end{abstract}

6 THEORETICAL BACKGROUND

7 Development and Validation of AIM-Q Scales

9 Measurement Validity of the Motive Scales

10 CURRENT STUDY

10 MATERIALS AND METHODS

10 Translation

10 Data Collection

11 Instrument

11 Samples

11 Statistical Analysis

13 RESULTS

13 Descriptive Statistics

16 Initial Models

16 Evaluating Measurement Invariance

17 DISCUSSION

19 REFERENCES

21 APPENDIX (LANGUAGE VERSIONS OF THE QUICK AIM 60-ITEM SCALE) 


\section{THEORETICAL BACKGROUND}

T

his is a study of factor invariance of a 15-dimensional individual differences model of human motivation. Such multidimensional theories of human motivation are rare, although their utility has been acknowledged (Baumeister, 2016). Bernard, Mills, Swenson, and Walsh (2005) proposed a multidimensional theory of individual differences in human motivation. According to this theory, motivated behavior is purposeful and solves individual fitness challenges - involving individual survival and reproduction - and inclusive fitness challenges - involving interpersonal and social relationships and progeny (see Dawkins, 1982; Hamilton, 1964; Tooby \& Cosmides, 1992). Bugental (2000) proposed that separate human neurohormonal systems evolved in order to overcome these challenges within different sized social domains. This led to consideration of the social domains in which more complex and inclusive fitness behaviors may operate, which include: Individual, Dyadic, Small Group, Large Group, and Very Large Group (see Bernard, 2012).

Evolution does not produce perfect universal adaptations; some individuals are better adapted to their environment. Therefore, individuals should vary in the strength of motive adaptations and this variation should allow motives to be measured as individual differences constructs. If, as Hogan (1996) contended, personality dispositions reflect reputation that influenced past survival and fitness, then perhaps motivational dispositions also reflect reputation that influenced them as well.

Bernard et al.'s (2005; Bernard, Mills, Swenson, \&Walsh, 2008; see also Bernard, 2012) action-trait (A-T) theory of human motivation conceptualized motivational dispositions as "traits of action" that operate within narrow domains of human behavior. A-T theory drew on Holt's (1931) and Tolman's (1932) "purposive psychology" and evolutionary principles suspected to drive the development of these predispositions. A-T theory offered specific criteria for the identification of a small set of independent, narrow domain motives which incorporated roles for emotion, culture, learning, conscious, and nonconscious processes, and tentatively proposed neuropsychological circuits presumed to underlie the traits of action (see Bernard, Mills, Swenson, \& Walsh, 2005). The multidimensionality of A-T theory contrasts with uni- or bi-dimensional approaches that are primarily cognitive, e.g., self-regulation (Oettingen, Bulgarella, Henderson, \& Gollwitzer, 2004) achievement motivation (McClelland, Atkinson, Clark, \& Lowell, 1976), and intrinsic-extrinsic motivation (Deci \& Ryan, 1987). The field could benefit from multidimensional theories that incorporate biological, behavioral, and environmental influences. Moreover, evolutionary theory offers a solid ground for motivational theory as it tackles the why of contemporary behavior and sets logical constraints on theory (Bernard et al., 2005). The theory posits 15 motives based on behaviors that solved specific fitness problems in specific environments. The theory also suggests specific brain regions that have evolved to mediate specific motives (Bernard et al., 2005). The utility of considering evolutionary motives lies in the strength to predict future behavior - normal and abnormal (Bernard, 2012,2014b). A longitudinal study showed that motive strength at Time 1 can predict a statistically significant proportion of the variance

\footnotetext{
${ }^{1}$ Purposive psychology was a teleological refinement of James' (1890) instinct theory and is defined as “... [a] systematic exposition of psychological facts and principles which emphasizes the ends to which all actions are directed and the needs or purposes which instincts serve for the organism" (Harriman, 1975, p. 157).
} 
in 21 of 28 health-related, self-indulgent, socially valued, and socially discordant behaviors at Time 2 (Bernard \& Lac, 2014a).

A-T theory is unique in its use of evolutionary principles to posit specific criteria for determining motive traits of action and in postulating an assessment strategy for motive dimensions. This assessment strategy is called the Assessment of Individual Motives - Questionnaire (AIM-Q; Bernard, 2013a).

\section{DEVELOPMENT AND VALIDATION OF AIM-Q SCALES}

After reviewing challenges humans probably encountered in different sized social groups in the past (e.g., Bugental, 2000; Kenrick, Li, \& Butner, 2003), Bernard and colleagues (2005) derived a short list of such challenges (see Table 1). To avoid lengthy lists of instincts, drives, and needs that have plagued prior multidimensional motivational theories (see Dunlap, 1919), specific criteria were used to identify each motive: (1) it must address a recurring challenge in humans' past; (2) it had the potential to affect survival and fitness; and (3) it can be demonstrated to operate as a relatively independent individual differences dimension of purposive behavior today. The result was the identification of 15 motives that emerged based on putative evolutionary processes. The labels for the motives and their conceptual definitions (in terms of behavior) are listed in Table 1.

Table 1. Recurring Challenges, Motive Labels, Conceptual Definitions, and Example Items

\begin{tabular}{|c|c|c|c|}
\hline Challenge & Motive & Conceptual Definition & Example Item \\
\hline \multicolumn{4}{|c|}{ Integrity Motives } \\
\hline \multirow[t]{4}{*}{$\begin{array}{l}\text { Identification of } \\
\text { environmental re- } \\
\text { sources and hazards }\end{array}$} & $\begin{array}{l}\text { Environmental } \\
\text { Inquisitiveness }\end{array}$ & $\begin{array}{l}\text { Use resources to explore the physical environment; evaluate } \\
\text { resource availability/hazards in new and different things, } \\
\text { places, and situations }\end{array}$ & $\begin{array}{l}\text { I explore unknown } \\
\text { places to understand } \\
\text { them better. }\end{array}$ \\
\hline & Illness Avoidance & Use resources to maintain bodily integrity and health & $\begin{array}{l}\text { I am very careful not } \\
\text { to touch anything } \\
\text { after I wash my hands } \\
\text { in public place. }\end{array}$ \\
\hline & Threat Avoidance & $\begin{array}{l}\text { Use resources to maintain personal safety; avoid challenges to } \\
\text { one's person and resources }\end{array}$ & $\begin{array}{l}\text { I spend a lot of effort } \\
\text { to avoid physical } \\
\text { threats of any kind. }\end{array}$ \\
\hline & $\begin{array}{l}\text { Interpersonal } \\
\text { Inquisitiveness }\end{array}$ & $\begin{array}{l}\text { Use resources to explore the social environment; test limits and } \\
\text { how others act, react, and interact; compare oneself to others }\end{array}$ & $\begin{array}{l}\text { I try to understand } \\
\text { others by playfully } \\
\text { teasing them to see } \\
\text { how they react. }\end{array}$ \\
\hline $\begin{array}{l}\text { Counteracting } \\
\text { despair over the } \\
\text { awareness of } \\
\text { mortality }\end{array}$ & Meaning & $\begin{array}{l}\text { Use resources to identify with, construct, and maintain } \\
\text { a philosophy, purpose, or rationalization for existence (and } \\
\text { non-existence) and attempts to arrive at an understanding } \\
\text { and peace with the presumed purpose of life }\end{array}$ & $\begin{array}{l}\text { Ilearn about various } \\
\text { religions or philoso- } \\
\text { phies to understand } \\
\text { the purpose of life } \\
\text { better. }\end{array}$ \\
\hline \multicolumn{4}{|c|}{ Competitive Motives } \\
\hline $\begin{array}{l}\text { Competing for } \\
\text { control of resources }\end{array}$ & Aggression & $\begin{array}{l}\text { Use resources challenge and intimidate others for control of } \\
\text { resources; approach challenges to one's person and resources } \\
\text { with combative and intimidating displays and actions }\end{array}$ & $\begin{array}{l}\text { I use physical threats } \\
\text { so that others know } \\
\text { not to get in my way. }\end{array}$ \\
\hline
\end{tabular}




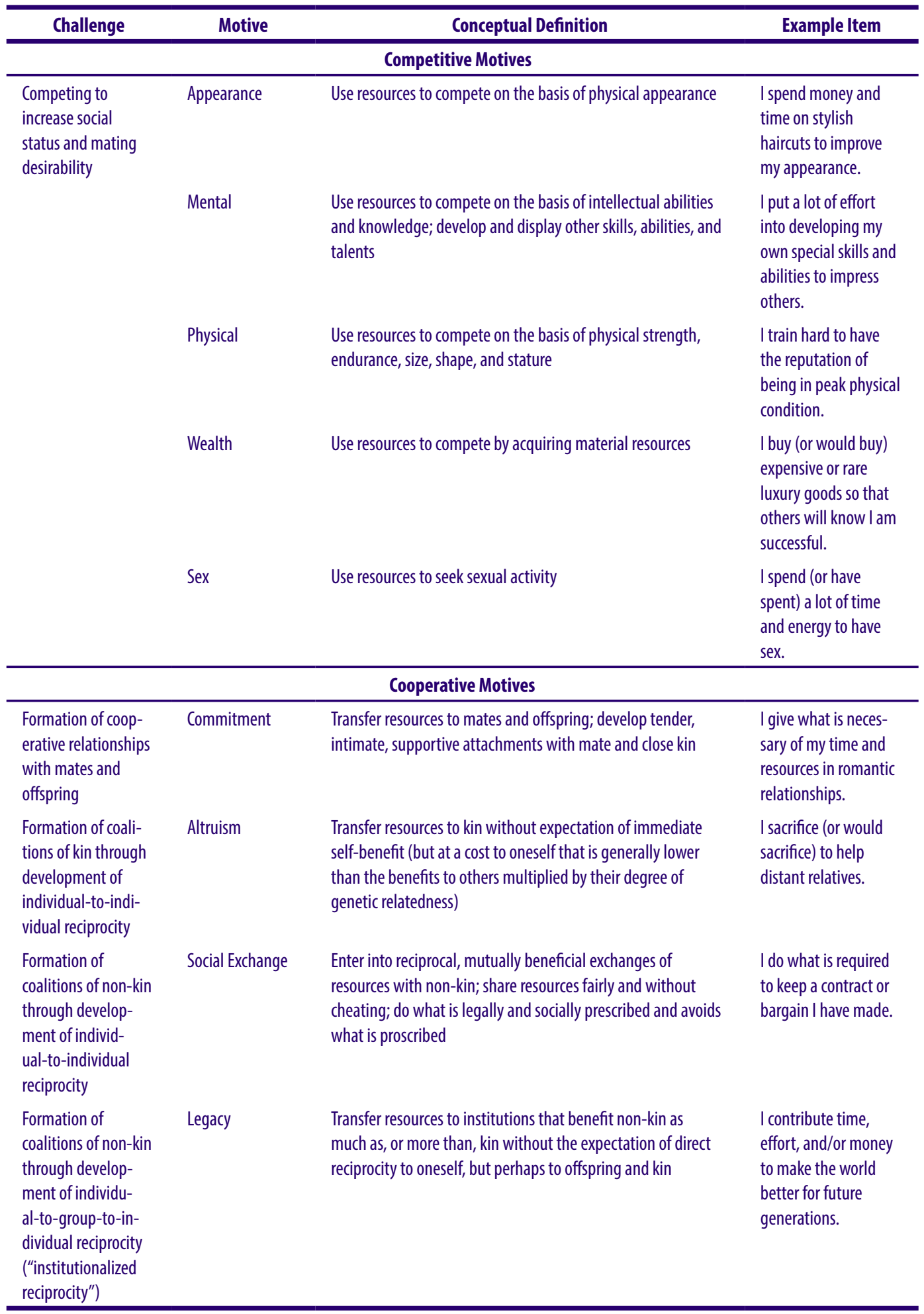

The conceptual definitions of the motive dimensions were used to generate an initial item pool of 180 items to assess the 15 motive factors ( 12 items per factor). The items in the factors were initially validated using Principal Components Analysis (Bernard et al., 2008). Furthermore, three preliminary versions of AIM-Q scales were constructed to permit a multitrait-multimethod (MTMM; Campbell \& Fiske, 1959) analysis of the convergent and discriminant validities of the motive factors across different assessment methods (Bernard et al., 2008). The MTMM matrix was scrutinized using Pearson correlations involving 
all 15 motive composites serving as the traits as applied to three methods that were completed by all participants. These three method types consisted of self-reports that varied based on instructional and response option modifications: (1) indicate the best use of your personal resources (responses ranging from strongly disagree to strongly agree); (2) endorse your own interests, desires, and likes (responses ranging from completely untrue to completely true); and (3) indicate your behavioral priorities (responses ranging from no importance at all to great importance). Results provided empirical support for the overall strategy, across the various measurement methods, of assessing individual differences in motivation with the AIM-Q motives.

The number of items was subsequently reduced to 120 in a revision that exhibited strong internal consistency reliabilities for each subscale. These subscales were demonstrated to be highly correlated with subscales from the original longer version (Bernard, 2013a). The next objective was to evaluate the factor structure of the 15 factors using confirmatory factor analysis and to reduce the inventory to an even more manageable length for participants (Bernard \& Lac, 2014a). Applying confirmatory factor analysis to the 120 -item variant of the scale, the model fit indices for the original 120 item scale were judged to be mediocre $(\mathrm{CFI}=.79, \mathrm{IFI}=.79, \mathrm{RMSEA}=.047)$. The inspection of post hoc modification indices revealed that poor model fit was attributed to items with substandard factor loadings. Accordingly, the four items with the highest standardized factor loadings for each factor were retained. This process yielded a 60-item 15-dimension version (named the "Quick AIM") that produced satisfactory fit indices (CFI $=.91, \mathrm{IFI}=.92$, and RMSEA $=.041$ ). Finally, using a different sample in the same study (Bernard \& Lac, 2014a), confirmatory factor analysis successfully cross-validated the factor structure and yielded desirable fit indices. Additional confirmatory factor analyses using this sample examined competing factor structures, including multidimensional variants of orthogonal (non-correlated factors) and oblique (correlated factors) models and a single-factor model (Bernard \& Lac, 2014a). Fit indices and factor loadings best supported the original, oblique 15-dimensional model. This version of the Quick AIM was used in the present study.

\section{MEASUREMENT VALIDITY OF THE MOTIVE SCALES}

Other reliability and validity studies have evaluated additional statistical properties of the AIM-Q motive scales (Bernard, 2013a) and supported the construct validity of AIM-Q and Quick AIM versions. First, AIM-Q scales have demonstrated convergent validity with reliable and valid external measures for aggression, need for cognition, playfulness, and sexuality (Bernard, 2007). Specifically, certain hypothesized motives correlated logically with measures of aggression (anger, hostility, physical aggression, verbal aggression) ranging from $r=.60$ to .67 ; measures of the need for cognition ranging from $r=-.54$ to .72 ; measures of playfulness (spontaneous, expressive, fun, creative, silly) ranging from $r=-.47$ to .62 ; and measures of sexuality ranging from $r=-.54$ to .47 .

Second, self-ratings on AIM-Q motives have been correlated with observer ratings by friends ( $r=.29$ to .64$)$ and relatives ( $r=.32$ to .56 ; Bernard, 2009). This suggests that self and other judgments of motives converge in terms of the interpretation and meaning of the motive dimensions as descriptors of behavior. Third, experimental research of the motives' validity demonstrated that differences in some motives can affect judgments of facial attractiveness (Bernard \& Hardy, 2014).

Fourth, A-T theory predicted that motives should explain variance in both normal and abnormal personality traits. Indeed, studies have suggested that certain motives are correlated with normal personality traits as assessed by the HEXACO (Lee \& Ashton, 2004) six factor model (Bernard, Lac, \& Lukaszewski, 2013) and the Five Factor Model 
of personality (Bernard, 2010). And, as predicted, certain motives are also correlated with abnormal personality traits such as borderline personality, psychopathy, and maladjustment (Bernard, 2013b).

Fifth, AIM-Q motives predicted a variety of self-reported behaviors confirmed by reports of observers (Bernard, 2009). These behaviors included smoking $\left(R_{\text {Adj. }}^{2}=.15\right)$, drinking alcohol $\left(R_{\text {Adj. }}^{2}=.21\right)$, using illegal substances $\left(R_{\text {Adj. }}^{2}=.17\right)$, holding positions of leader$\operatorname{ship}\left(R_{\text {Adj. }}^{2}=.29\right)$, and exercising $\left(R_{\text {Adj. }}^{2}=.42\right)$. Finally, a longitudinal study to address the issue of predictive validity demonstrated that AIM-Q motives were incrementally valid predictors of future behaviors (Bernard \& Lac, 2014b).

\section{CURRENT STUDY}

This programmatic series of studies have supported the measurement validity of the AIM-Q and Quick AIM scales up to this point. However, until now, all previous studies have used only U.S. English-speaking samples. It is currently unknown whether results would be similar if the research had been conducted with other cultures or languages. Thus, this is the first study to attempt to corroborate the 15 -factor structure of motive dimensions across linguistic and cultural groups. For the theoretical framework and scale to be linguistically invariant, the individual differences in motive dimensions should not be unique to the U.S. English speaking samples. Thus, the present study was meant to examine the factor structure and measurement invariance (configural, metric, and scalar) of the 15 Quick AIM scales cross-linguistically for the first time and is a crucial step in theory development and hypothesis testing.

\section{MATERIALS AND METHODS}

\section{TRANSLATION}

The U.S. English Quick AIM was translated into four different languages: Croatian, Polish, German and French. The International Testing Commission (2008) guidelines were followed for the translations of the original U.S. English Quick AIM items and instructions. Bilingual doctoral level psychologists and graduate students participated in multiple forward and backward translations. The back translations into English were reviewed by Quick AIM author (L. Bernard). Edits were provided as feedback to translators who modified items as necessary. Modified items were once again back translated into English for a final check. All the language versions used in the current study are in the Appendix.

\section{DATA COLLECTION}

Participants were treated in accordance with the ethical standards of institutional and governmental organizations for the treatment of human participants. All administrations were performed anonymously, with minimal demographic data collected. The Quick AIM was administered online through Internet survey programs and took approximately 15 minutes to complete. 


\section{INSTRUMENT}

Quick AIM. The Quick AIM is a U.S. English language 60-item (Bernard, 2013a; Bernard \& Lac, 2014a) empirically validated version of the AIM-Q scales. Each factor consists of 4 items. Respondents endorsed each item as true or untrue using a 6-point scale: 1 (completely untrue), 2 (mostly untrue), 3 (slightly untrue), 4 (slightly true), 5 (mostly true), to 6 (completely true). The Quick AIM scales' internal consistency reliabilities obtained in the current study are listed in Table 2.

\section{SAMPLES}

Samples from five languages were recruited - Croatian, French, German, Polish, and English:

Croatian. The sample consisted of $N=614$ participants ranging in age from 16 to 73 years $(M=28.0, S D=9.7)$. Fifty-seven percent of the sample was female. Half of the sample were university students in Croatia studying psychology and half were non-students recruited by the students.

French. The sample consisted of $N=246$ participants studying psychology in Switzerland. Due to local privacy concerns, data about age and sex were not obtained.

German. The sample consisted of $N=154$ participants ranging in age from 18 to 57 years $(M=26.0, S D=7.8)$. Sixty-seven percent of the sample was female. The sample was comprised of university students studying psychology in Switzerland.

Polish. The sample consisted of $N=314$ participants ranging in age from 19 to 71 years $(M=31.5, S D=12.7)$. Sixty-five percent of the sample was female. The majority of the sample was university students studying psychology in Poland.

U.S. English. The sample consisted of $N=490$ participants ranging in age from 18 to 78 years $(M=27.7, S D=13.50)$. One-third of the participants were psychology university students and two-thirds were non-student adults living in diverse geographic regions, mostly within California. Fifty-six percent of the sample was female.

\section{STATISTICAL ANALYSIS}

The skewness of the 60 items ranged from -0.83 to 2.87 for the Croatian sample, -2.03 to 2.06 for the French sample, -1.65 to 2.57 for the German sample, -1.24 to 2.23 for the Polish sample, and -1.55 to 1.42 for the U.S. sample. Thus, the items were found to be within reasonable boundaries of normality and therefore the data were appropriate for factor-analytic techniques (Tabachnick \& Fidell, 2013; Warner, 2013). However, robust estimation will be applied to the statistical techniques to automatically correct for any departures from normality (Hoyle, 2012).

Considering that 15 factors will be estimated in the study, model testing with exploratory structural equation modeling (ESEM) was warranted. This approach represents the integration of exploratory factor analysis (EFA) and confirmatory factor analysis (CFA). EFA is not conducive for testing measurement and structural invariance across groups, while CFA requires that each item is specified to load exclusively on its dominant factor and constrained to exactly zero loadings on all other factors. Thus, CFA has been criticized as overly restrictive and not reflecting the reality of the data. Previous investigations scrutinizing multi-factor structures, as the Big Five personality inventory, have suggested that ESEM yields better approximation of the underlying data (Marsh et al., 2010; Vazsonyi, Ksinan, Mikuška, \& Jiskrova, 2015). Marsh, Morin, Parker, and Kaur (2014) stated it is almost impossible to generate an acceptable model fit in CFA when multiple factors (5 to 10$)$ are 
measured by a reasonable number of items ( 5 to 10$)$ per subscale in an inventory of at least 50 items. The number of factors and total number of items in this investigation exceeds this recommendation and therefore indicates that ESEM would be the ideal technique.

ESEM was conducted using Mplus 7.13 (Muthén \& Muthén, 2012). Loadings of items intended to measure a given factor were freely estimated while all cross-loadings were allowed and targeted to be close to zero using "target rotation" (Marsh, Morin, Parker, \& Kaur, 2014; Morin, Boudrias, Marsh, Madore, \& Desrumaux, 2016). Marsh and colleagues (2014) state that "target rotation is particularly appropriate when there is a clearly defined a priori factor structure and a reasonable approximation to simple structure" and that this rotation is "based on partial knowledge of the factor structure, and is consistent with the view that ESEM is more typically used for confirmatory rather than exploratory purposes" (Marsh et al., 2014). Thus, ESEM using target rotation was pursued given that we possess partial knowledge of the factor structure from previous studies using the English version (but not the other translated versions) and that the research purpose was to confirm the factor structure across the other languages. Consistent with ESEM, the 15 factors were permitted to be correlated. The estimator was maximum likelihood estimation with robust estimation to account for variable departures from normality. A model was estimated for each linguistic sample.

After obtaining acceptable factor structures for each linguistic group, multi-group analyses were conducted using recommended guidelines (Byrne, 2012; Davidov, Meuleman, Cieciuch, Schmidt, \& Billiet, 2014; Lance \& Vandenberg, 2002) to test for measurement invariance of the questionnaire. Three levels of invariance were tested: Configural, metric and scalar. Configural invariance requires that latent factors are loaded by the same items in all groups, with each group representing a different language. Metric invariance requires that factor loadings of the items on the latent factors are the same across groups. Scalar invariance requires that intercepts of all items are equal across groups, in addition to the equality constraint of loadings at the metric level. Metric invariance is a precondition for meaningful comparisons of factor correlations across groups and scalar invariance is a precondition of latent means comparison across groups (Chen, 2007; Lance \& Vandenberg, 2002). Byrne, Shavelson, and Muthén (1989) introduced the concept of partial measurement invariance. It implies that at least two parameters (loadings at the metric level or loadings and intercepts at the scalar level) are found to be equal across groups, while other parameters are freely estimated. Partial measurement invariance is assumed to allow the same types of comparisons as full measurement invariance (Byrne, Shavelson, \& Muthén, 1989).

Several fit indices were used to scrutinize the overall fit of models. The model chi-square test was reported. Goodness-of-fit values of the Comparative Fit Index (CFI) ranged from 0.00 to 1.00 , with higher values signifying a better fitting model (Tabachnick \& Fidell, 2007). A value above .90 indicates good fit and above .95 very good fit ( $\mathrm{Hu} \&$ Bentler, 1999). Two badness-of-fit indices were also evaluated. The Root Mean-Square Error of Approximation (RMSEA) is adequately sensitive in detecting model misspecification and offers appropriate information about model quality (MacCallum \& Austin, 2000). A value below .05 indicates close fit, between .05 and .08 fair fit, between .08 and .10 mediocre fit, and above .10 poor fit (MacCallum, Browne, \& Sugawara, 1996). According to Hu and Bentler (1999) RMSEA below .08 indicates a good model fit. Standardized Root Mean-Square Residual (SRMR) has shown to be useful for detecting model misspecification, with a recommended value below .08 diagnostic of good fit (Hu \& Bentler, 1998).

In evaluating the extent that measurement invariance was established, we applied two criteria: the global fit proposed by Chen (2007) and the examination of local misspecification as proposed by Saris, Satorra, and van der Veld (2009) and applied to the test of measurement invariance by Cieciuch, Davidov, Oberski, and Algesheimer (2015). We used the first approach to test for the full measurement invariance and the second for 
partial measurement invariance to identify noninvariant loadings and intercepts. For the first approach, metric noninvariance is indicated by a change larger than .010 in CFI, supplemented by a change larger than .015 in RMSEA or a change larger than .030 in SRMR compared with the configural invariance model. Regarding scalar invariance, noninvariance is indicated by a change larger than .010 in CFI, supplemented by a change larger than .015 in RMSEA or a change larger than .010 in SRMR compared with the metric invariance model. For the second approach, power of the test was taken into account and local misspecification was examined (Cieciuch, Davidov, Oberski, \& Algesheimer, 2015). This way, we could identify parameters with noninvariant loadings and intercepts. We followed the suggestion of Saris et al. (2009) and treated deviation in loadings greater than .40 and in intercepts greater than .10 as a sign of serious misspecification.

\section{RESULTS}

\section{DESCRIPTIVE STATISTICS}

Descriptive statistics are presented in Tables 2-7. Table 2 presents means, standard deviations and Cronbach's alpha coefficients for the scales in each group and Table 3-7 present intercorrelations between scales in each country.

Table 2. Cronbach's Alpha for Quick AIM

\begin{tabular}{|c|c|c|c|c|c|c|c|c|c|c|}
\hline & \multicolumn{2}{|c|}{ English (U.S.) } & \multicolumn{2}{|r|}{ Croatian } & \multicolumn{2}{|r|}{ German } & \multicolumn{2}{|r|}{ Polish } & \multicolumn{2}{|r|}{ French } \\
\hline & a & $\begin{array}{c}M \\
(S D)\end{array}$ & a & $\begin{array}{c}M \\
(S D)\end{array}$ & a & $\begin{array}{c}M \\
(S D)\end{array}$ & $a$ & $\begin{array}{c}M \\
(S D)\end{array}$ & a & $\begin{array}{c}M \\
(S D)\end{array}$ \\
\hline Aggression & .86 & $2.27(1.19)$ & .76 & $1.61(0.77)$ & .86 & $1.67(0.88)$ & .80 & $1.60(0.73)$ & .72 & $1.88(0.82)$ \\
\hline Threat Avoidance & .69 & $3.98(1.01)$ & .68 & $3.91(1.01)$ & .53 & $3.43(0.91)$ & .59 & $3.35(0.89)$ & .63 & $3.67(0.92)$ \\
\hline Illness Avoidance & .69 & $3.45(1.09)$ & .59 & $3.12(0.96)$ & .67 & $2.82(1.09)$ & .52 & $3.17(0.90)$ & .60 & $2.90(0.97)$ \\
\hline $\begin{array}{l}\text { Environment } \\
\text { Inquisitiveness }\end{array}$ & .80 & $4.40(0.93)$ & .87 & $4.47(1.02)$ & .83 & $4.81(0.87)$ & .69 & $4.16(0.86)$ & .83 & $4.73(0.82)$ \\
\hline $\begin{array}{l}\text { Interpersonal } \\
\text { Inquisitiveness }\end{array}$ & .87 & $3.27(1.30)$ & .91 & $2.43(1.24)$ & .87 & $3.07(1.25)$ & .88 & $2.38(1.21)$ & .88 & $3.73(1.19)$ \\
\hline Sex & .86 & $2.33(1.30)$ & .86 & $2.03(1.05)$ & .84 & $2.47(1.17)$ & .79 & $2.24(1.06)$ & .91 & $2.30(1.28)$ \\
\hline Appearance & .84 & $3.28(1.34)$ & .79 & $1.69(0.87)$ & .88 & $2.57(1.19)$ & .87 & $2.46(1.26)$ & .81 & $2.69(1.18)$ \\
\hline Wealth & .87 & $2.93(1.28)$ & .83 & $1.62(0.84)$ & .86 & $1.83(0.97)$ & .90 & $2.19(1.18)$ & .88 & $2.09(1.07)$ \\
\hline Mental & .81 & $3.40(1.12)$ & .84 & $2.56(1.14)$ & .77 & $3.45(1.06)$ & .84 & $2.94(1.17)$ & .85 & 3.07 (1.16) \\
\hline Physical & .91 & $3.48(1.40)$ & .88 & $2.59(1.25)$ & .92 & $2.95(1.35)$ & .92 & 2.54 (1.24) & .74 & $3.14(1.11)$ \\
\hline Commitment & .88 & $4.95(1.01)$ & .89 & $4.56(1.16)$ & .80 & $4.74(0.98)$ & .77 & $4.68(0.97)$ & .72 & $4.96(0.77)$ \\
\hline Altruism & .82 & $4.45(1.00)$ & .74 & $3.98(0.98)$ & .84 & $4.32(1.03)$ & .81 & $4.25(0.90)$ & .81 & $4.49(0.94)$ \\
\hline Social Exchange & .77 & $5.15(0.71)$ & .72 & $5.11(0.63)$ & .73 & $5.38(0.52)$ & .71 & $4.80(0.66)$ & .74 & $5.13(0.61)$ \\
\hline Legacy & .84 & $3.88(1.15)$ & .87 & $3.20(1.24)$ & .85 & $3.35(1.22)$ & .85 & $3.17(1.11)$ & .91 & 3.14 (1.27) \\
\hline Meaning & .89 & $3.49(1.25)$ & .92 & $2.99(1.42)$ & .90 & $3.75(1.28)$ & .92 & $2.74(1.26)$ & .91 & $3.46(1.32)$ \\
\hline
\end{tabular}


Table 3. Intercorrelations between Quick AIM scales in Croatia

\begin{tabular}{|c|c|c|c|c|c|c|c|c|c|c|c|c|c|c|}
\hline & (1) & (2) & (3) & (4) & (5) & (6) & (7) & (8) & (9) & (10) & (11) & (12) & (13) & (14) \\
\hline \multicolumn{15}{|l|}{ (1) Aggression } \\
\hline $\begin{array}{l}\text { (2) Threat } \\
\text { Avoidance }\end{array}$ & $-.22^{* *}$ & & & & & & & & & & & & & \\
\hline $\begin{array}{l}\text { (3) Illness } \\
\text { Avoidance }\end{array}$ & $.10^{*}$ & $.29^{* *}$ & & & & & & & & & & & & \\
\hline $\begin{array}{l}\text { (4) Environment } \\
\text { Inquisitiveness }\end{array}$ & -.03 & .05 & -.01 & & & & & & & & & & & \\
\hline $\begin{array}{l}\text { (5) Interpersonal } \\
\text { Inquisitiveness }\end{array}$ & $.42^{* *}$ & $-.13^{* *}$ & .03 & $.11^{* *}$ & & & & & & & & & & \\
\hline (6) Sex & $.34^{* *}$ & $-.14^{* *}$ & .00 & -.04 & $.37^{* *}$ & & & & & & & & & \\
\hline (7) Appearance & $.17^{* *}$ & -.06 & $.09^{*}$ & $.09^{*}$ & $.11^{* *}$ & $.15^{* *}$ & & & & & & & & \\
\hline (8) Wealth & $.36^{* *}$ & $-.11^{* *}$ & $.09^{*}$ & -.03 & $.27^{* *}$ & $.32^{* *}$ & $.65^{* *}$ & & & & & & & \\
\hline (9) Mental & $.35^{* *}$ & .01 & .06 & $.11^{* * *}$ & $.41^{* *}$ & $.28^{* * *}$ & $.28^{* *}$ & $.49^{* *}$ & & & & & & \\
\hline (10) Physical & $.30^{* * *}$ & $-.10^{*}$ & $.12^{* *}$ & $.09^{*}$ & $.16^{* * *}$ & $.28^{* * *}$ & $.25^{* *}$ & $.33^{* *}$ & $.39^{* *}$ & & & & & \\
\hline (11) Commitment & $-.12^{* *}$ & $.16^{* *}$ & .03 & $.16^{* *}$ & -.04 & -.06 & .02 & $-.12^{* *}$ & -.02 & .02 & & & & \\
\hline (12) Altruism & -.04 & .07 & .00 & $.19^{* *}$ & .02 & .01 & -.04 & -.02 & .05 & .08 & $.25^{* *}$ & & & \\
\hline $\begin{array}{l}\text { (13) Social } \\
\text { Exchange }\end{array}$ & $-.21^{* *}$ & $.24^{* *}$ & $.12^{* *}$ & $.34^{* * *}$ & $-.15^{* *}$ & $-.18^{* * *}$ & $-.13^{* *}$ & $-.24^{* *}$ & -.04 & .01 & $.33^{* *}$ & $.34^{* *}$ & & \\
\hline (14) Legacy & -.06 & $.09^{*}$ & .06 & $.26^{* *}$ & $-.13^{* *}$ & $-.15^{* *}$ & .04 & -.06 & .00 & .00 & .05 & $.31^{* *}$ & $.30^{* *}$ & \\
\hline (15) Meaning & $.14^{* *}$ & -.06 & -.02 & $.35^{* *}$ & $.13^{* * *}$ & $.08^{*}$ & .03 & -.03 & $.25^{* *}$ & .07 & .07 & $.11^{* *}$ & $.11^{* *}$ & $.29^{*}$ \\
\hline
\end{tabular}

Table 4. Intercorrelations between Quick AIM scales in French

\begin{tabular}{|c|c|c|c|c|c|c|c|c|c|c|c|c|c|c|}
\hline & (1) & (2) & (3) & (4) & (5) & (6) & (7) & (8) & (9) & (10) & (11) & (12) & (13) & (14) \\
\hline \multicolumn{15}{|l|}{ (1) Aggression } \\
\hline $\begin{array}{l}\text { (2) Threat } \\
\text { Avoidance }\end{array}$ & -.09 & & & & & & & & & & & & & \\
\hline $\begin{array}{l}\text { (3) Illness } \\
\text { Avoidance }\end{array}$ & .06 & $.30^{* *}$ & & & & & & & & & & & & \\
\hline $\begin{array}{l}\text { (4) Environment } \\
\text { Inquisitiveness }\end{array}$ & .03 & -.07 & .00 & & & & & & & & & & & \\
\hline $\begin{array}{l}\text { (5) Interpersonal } \\
\text { Inquisitiveness }\end{array}$ & $.40^{* *}$ & -.08 & -.01 & .07 & & & & & & & & & & \\
\hline (6) Sex & $.39^{* *}$ & .01 & .02 & -.04 & $.32^{* *}$ & & & & & & & & & \\
\hline (7) Appearance & $.26^{* *}$ & $.14^{*}$ & $.16^{*}$ & .05 & $.15^{*}$ & $.23^{* *}$ & & & & & & & & \\
\hline (8) Wealth & $.39^{* *}$ & $.18^{* *}$ & $.18^{* *}$ & -.04 & $.27^{* *}$ & $.37^{* *}$ & $.57^{* *}$ & & & & & & & \\
\hline (9) Mental & $.41^{* *}$ & .10 & .03 & $.21^{* *}$ & $.24^{* *}$ & $.42^{* *}$ & $.34^{* *}$ & $.43^{* *}$ & & & & & & \\
\hline (10) Physical & $.25^{* * *}$ & .00 & -.03 & $.17^{* *}$ & $.14^{*}$ & $.28^{* *}$ & $.31^{* *}$ & $.25^{* *}$ & $.45^{* *}$ & & & & & \\
\hline (11) Commitment & .03 & .04 & -.04 & $.13^{*}$ & .04 & .01 & $.20^{* *}$ & .08 & $.18^{* *}$ & $.21^{* *}$ & & & & \\
\hline (12) Altruism & -.06 & -.03 & .01 & $.16^{*}$ & .00 & -.07 & .07 & -.03 & -.01 & .10 & $.15^{*}$ & & & \\
\hline $\begin{array}{l}\text { (13) Social } \\
\text { Exchange }\end{array}$ & $-.32^{* *}$ & .05 & -.05 & $.27^{* *}$ & $-.20^{* *}$ & $-.33^{* *}$ & .05 & $-.24^{* *}$ & -.09 & -.04 & $.27^{* *}$ & $.32^{* *}$ & & \\
\hline (14) Legacy & .09 & .01 & .12 & $.33^{* *}$ & -.02 & -.02 & .01 & -.11 & .09 & $.16^{*}$ & .01 & $.25^{* *}$ & $.22^{* * *}$ & \\
\hline (15) Meaning & $.22^{* *}$ & -.04 & .08 & $.36^{* *}$ & .08 & $.13^{*}$ & .00 & .00 & $.31^{* *}$ & $.31^{* *}$ & .08 & $.15^{*}$ & .07 & $.36^{* *}$ \\
\hline
\end{tabular}

Note. ${ }^{*} p<.05 ;{ }^{* *} p<.01$ 
Table 5. Intercorrelations between Quick AIM scales in Germany

\begin{tabular}{|c|c|c|c|c|c|c|c|c|c|c|c|c|c|c|}
\hline & (1) & (2) & (3) & (4) & (5) & (6) & (7) & (8) & (9) & (10) & (11) & (12) & (13) & (14) \\
\hline \multicolumn{15}{|l|}{ (1) Aggression } \\
\hline $\begin{array}{l}\text { (2) Threat } \\
\text { Avoidance }\end{array}$ & .01 & & & & & & & & & & & & & \\
\hline $\begin{array}{l}\text { (3) Illness } \\
\text { Avoidance }\end{array}$ & $.22^{* *}$ & $.18^{*}$ & & & & & & & & & & & & \\
\hline $\begin{array}{l}\text { (4) Environment } \\
\text { Inquisitiveness }\end{array}$ & .01 & -.13 & .16 & & & & & & & & & & & \\
\hline $\begin{array}{l}\text { (5) Interpersonal } \\
\text { Inquisitiveness }\end{array}$ & $.46^{* *}$ & -.04 & $.20^{*}$ & $.20^{*}$ & & & & & & & & & & \\
\hline (6) Sex & $.44^{* *}$ & .07 & $.17^{*}$ & .13 & $.43^{* *}$ & & & & & & & & & \\
\hline (7) Appearance & $.31^{* *}$ & -.02 & $.27^{* *}$ & .09 & $.20^{*}$ & $.27^{* *}$ & & & & & & & & \\
\hline (8) Wealth & $.48^{* *}$ & .10 & $.21^{* *}$ & .04 & $.21^{*}$ & $.32^{* *}$ & $.72^{* *}$ & & & & & & & \\
\hline (9) Mental & $.33^{* *}$ & .08 & .08 & $.16^{*}$ & $.31^{* *}$ & $.34^{* *}$ & $.36^{* *}$ & $.44^{* *}$ & & & & & & \\
\hline (10) Physical & $.43^{* *}$ & .04 & $.23^{* *}$ & $.17^{*}$ & $.36^{* *}$ & $.45^{* *}$ & $.32^{* *}$ & $.39^{* *}$ & $.47^{* *}$ & & & & & \\
\hline (11) Commitment & .02 & .07 & $.16^{*}$ & .11 & -.02 & .09 & -.04 & -.02 & $.19^{*}$ & .12 & & & & \\
\hline (12) Altruism & $.19^{*}$ & -.09 & -.05 & $.24^{* *}$ & $.23^{* *}$ & .08 & .08 & .13 & .13 & .13 & .13 & & & \\
\hline $\begin{array}{l}\text { (13) Social } \\
\text { Exchange }\end{array}$ & $-.19^{*}$ & -.08 & .03 & $.27^{* *}$ & -.14 & $-.24^{* *}$ & .06 & -.01 & .08 & -.01 & $.29^{* *}$ & $.25^{* *}$ & & \\
\hline (14) Legacy & -.03 & .08 & .05 & $.25^{* *}$ & -.01 & -.09 & -.06 & -.08 & .06 & -.01 & .08 & .08 & $.20^{*}$ & \\
\hline (15) Meaning & .13 & .09 & $.17^{*}$ & $.48^{* *}$ & .13 & $.18^{*}$ & .04 & .06 & $.19^{*}$ & .09 & .02 & .13 & $.17^{*}$ & $.34^{* *}$ \\
\hline
\end{tabular}

Table 6. Intercorrelations between Quick AIM scales in Poland

\begin{tabular}{|c|c|c|c|c|c|c|c|c|c|c|c|c|c|c|}
\hline & (1) & (2) & (3) & (4) & (5) & (6) & (7) & (8) & (9) & (10) & (11) & (12) & (13) & (14) \\
\hline \multicolumn{15}{|l|}{ (1) Aggression } \\
\hline $\begin{array}{l}\text { (2) Threat } \\
\text { Avoidance }\end{array}$ & .03 & & & & & & & & & & & & & \\
\hline $\begin{array}{l}\text { (3) Illness } \\
\text { Avoidance }\end{array}$ & $.12^{*}$ & $.36^{* *}$ & & & & & & & & & & & & \\
\hline $\begin{array}{l}\text { (4) Environment } \\
\text { Inquisitiveness }\end{array}$ & -.03 & $.17^{* *}$ & $.25^{* *}$ & & & & & & & & & & & \\
\hline $\begin{array}{l}\text { (5) Interpersonal } \\
\text { Inquisitiveness }\end{array}$ & $.44^{* *}$ & -.08 & -.04 & $.23^{* *}$ & & & & & & & & & & \\
\hline (6) Sex & $.39^{* *}$ & .03 & .06 & $.18^{* *}$ & $.41^{* *}$ & & & & & & & & & \\
\hline (7) Appearance & $.23^{* *}$ & .08 & $.25^{* *}$ & $.18^{* *}$ & $.18^{* *}$ & $.40^{* * *}$ & & & & & & & & \\
\hline (8) Wealth & $.41^{* *}$ & .03 & $.15^{* *}$ & $.14^{*}$ & $.36^{* *}$ & $.48^{* *}$ & $.74^{* *}$ & & & & & & & \\
\hline (9) Mental & $.37^{* *}$ & $.19^{* *}$ & $.13^{*}$ & $.39^{* *}$ & $.40^{* * *}$ & $.43^{* *}$ & $.47^{* *}$ & $.61^{* *}$ & & & & & & \\
\hline (10) Physical & $.33^{* *}$ & .03 & $.18^{* * *}$ & $.20^{* * *}$ & $.25^{* *}$ & $.42^{* *}$ & $.47^{* *}$ & $.45^{* *}$ & $.50^{* *}$ & & & & & \\
\hline (11) Commitment & -.10 & $.13^{*}$ & .05 & $.22^{* *}$ & .04 & $.12^{*}$ & $.13^{*}$ & .10 & .11 & .05 & & & & \\
\hline (12) Altruism & $-.14^{*}$ & .06 & .05 & $.16^{* *}$ & -.08 & -.10 & .02 & -.08 & .00 & $.11^{*}$ & $.36^{* *}$ & & & \\
\hline $\begin{array}{l}\text { (13) Social } \\
\text { Exchange }\end{array}$ & $-.34^{* *}$ & $.15^{* *}$ & .05 & $.18^{* *}$ & $-.22^{* *}$ & $-.21^{* *}$ & -.11 & $-.22^{* *}$ & -.07 & $-.13^{*}$ & $.31^{* *}$ & $.47^{* *}$ & & \\
\hline (14) Legacy & .02 & .10 & $.29^{* * *}$ & .07 & $-.15^{* *}$ & -.07 & .11 & -.01 & -.05 & .08 & $.16^{* *}$ & $.41^{* *}$ & $.30^{* * *}$ & \\
\hline (15) Meaning & $.19^{* *}$ & .04 & .05 & $.429^{* *}$ & $.23^{* *}$ & $.23^{* * *}$ & $.14^{*}$ & .10 & $.24^{* *}$ & $.21^{* *}$ & .11 & $.14^{*}$ & .03 & $.31^{* *}$ \\
\hline
\end{tabular}

Note. ${ }^{*} p<.05 ; * *$ $p<.01$ 
Table 7. Intercorrelations between Quick AIM scales in U.S.

\begin{tabular}{|c|c|c|c|c|c|c|c|c|c|c|c|c|c|c|}
\hline & (1) & (2) & (3) & (4) & (5) & (6) & (7) & (8) & (9) & (10) & (11) & (12) & (13) & (14) \\
\hline \multicolumn{15}{|l|}{ (1) Aggression } \\
\hline $\begin{array}{l}\text { (2) Threat } \\
\text { Avoidance }\end{array}$ & $-.24^{* *}$ & & & & & & & & & & & & & \\
\hline $\begin{array}{l}\text { (3) Illness } \\
\text { Avoidance }\end{array}$ & .03 & $.34^{* *}$ & & & & & & & & & & & & \\
\hline $\begin{array}{l}\text { (4) Environment } \\
\text { Inquisitiveness }\end{array}$ & -.05 & .02 & -.01 & & & & & & & & & & & \\
\hline $\begin{array}{l}\text { (5) Interpersonal } \\
\text { Inquisitiveness }\end{array}$ & $.49^{* *}$ & $-.13^{* *}$ & -.08 & $.11^{*}$ & & & & & & & & & & \\
\hline (6) Sex & $.51^{* *}$ & $-.19^{* *}$ & $-.16^{* *}$ & .05 & $.36^{* *}$ & & & & & & & & & \\
\hline (7) Appearance & $.19^{* * *}$ & $.19^{* *}$ & $.12^{* *}$ & .05 & .03 & $.18^{* *}$ & & & & & & & & \\
\hline (8) Wealth & $.47^{* *}$ & .05 & .05 & .02 & $.30^{* *}$ & $.35^{* *}$ & $.63^{* *}$ & & & & & & & \\
\hline (9) Mental & $.45^{* *}$ & -0.04 & -.01 & $.18^{* *}$ & $.41^{* *}$ & $.36 *$ & $.32^{* *}$ & $.55^{* *}$ & & & & & & \\
\hline (10) Physical & $.40^{* *}$ & -.05 & .08 & .08 & $.26 *$ & $.28^{* *}$ & $.27^{* * *}$ & $.42^{* *}$ & $.50^{* *}$ & & & & & \\
\hline (11) Commitment & $-.24^{* * *}$ & $.13^{* *}$ & .04 & $.32^{* *}$ & -.03 & -.06 & $.14^{* *}$ & .01 & .05 & .06 & & & & \\
\hline (12) Altruism & $-.10^{*}$ & .04 & $.10^{*}$ & $.30^{* *}$ & .01 & .00 & -.01 & -.06 & $.13^{* * *}$ & $.17^{* *}$ & $.30^{* *}$ & & & \\
\hline $\begin{array}{l}\text { (13) Social } \\
\text { Exchange }\end{array}$ & $-.45^{* *}$ & $.18^{* *}$ & .08 & $.32^{* *}$ & $-.24^{* *}$ & $-.32^{* *}$ & $-.10^{*}$ & $-.23^{* *}$ & $-.09^{*}$ & -.07 & $.46^{* *}$ & $.37^{* *}$ & & \\
\hline (14) Legacy & -.08 & $.18^{* *}$ & $.21^{* *}$ & $.28^{* *}$ & $-.15^{* *}$ & $-.14^{* *}$ & $.13^{* *}$ & -.03 & .04 & .04 & $.18^{* *}$ & $.23^{* *}$ & $.24^{* *}$ & \\
\hline (15) Meaning & $.11^{*}$ & .02 & .07 & $.42^{* *}$ & $.11^{*}$ & $.12^{* *}$ & .05 & .04 & $.22^{* *}$ & $.22^{* *}$ & $.18^{* *}$ & $.22^{* *}$ & $.13^{* *}$ & $.36^{* *}$ \\
\hline
\end{tabular}

\section{INITIAL MODELS}

The 15-factor structure representing the 60-item Quick AIM was separately estimated for each of the five samples using ESEM. All the items were retained in all single-group models. Fit indices for all the linguistic groups were desirable: CFI ranged from .924 to .965 , RMSEA from .033 to .051, and SRMR from .017 to .026 (see Table 8). Compared to the models for the other linguistic groups, the U. S. sample generated the best fit, not surprisingly, as the seminal version of the scale was developed in English. The factor structures for Croatian, German, and Polish also rendered acceptable fit indices. Although the software reported fit indices for French, convergence problems were indicated, so this structure should be interpreted with caution.

\section{EVALUATING MEASUREMENT INVARIANCE}

Due to estimation problems with the factor structure for French, this linguistic group was omitted from all subsequent analyses and multigroup ESEM was conducted using the U.S., Croatian, German, and Polish samples. No other problems were encountered in subsequent analyses. The multiple-group invariance tests for the four remaining languages indicated that item \#52 (see Appendix for item wording) should be dropped, resulting in a 59-item model. Furthermore, the pair of error terms involving items \#21 and \#51; and items \#41 and $\# 56$ were indicated to be strongly correlated, but these items were retained. 
Table 8. Exploratory Structural Equation Modeling of the Quick AIM 60-Item Scale

\begin{tabular}{lcccc}
\multicolumn{1}{c}{ Language } & Model $\chi^{2}$ & CFI & RMSEA [90\% Cl] & SRMR \\
\hline Croatian & 1876.55 & .952 & $.039[.036-.041]$ & .019 \\
\hline French & 1419.84 & .944 & $.043[.038-.048]$ & .023 \\
\hline German & 1369.63 & .924 & $.051[.045-.057]$ & .026 \\
\hline Polish & 1617.03 & .936 & $.046[.042-.050]$ & .022 \\
\hline English (U.S.) & 1505.94 & .965 & $.033[.030-.037]$ & .017 \\
\hline
\end{tabular}

Note. $d f=975$, all model chi-square tests were significant, $p<.01$.

Table 9. Global Fit Measures for the Multigroup Exploratory Structural Equation Modeling

\begin{tabular}{lccccc}
\hline \multicolumn{1}{c}{ Level of invariance } & Model $\mathbf{X}^{2}$ & $\mathrm{df}$ & RMSEA & SRMR & CFI \\
\hline Configural & 5871.57 & 3716 & $.038[.037-.040]$ & .019 & .955 \\
\hline Metric & 9538.45 & 5696 & $.041[.040-.043]$ & .045 & .920 \\
\hline Scalar & 10573.71 & 5828 & $.046[.044-.047]$ & .046 & .901 \\
\hline
\end{tabular}

Note. Quick AIM with item \#52 deleted. Two pairs of errors were correlated: Items \#21 and \#51, and items \#41 and \#56, but retained in the analysis. The French sample was not included.

The nested multi-group models involving the evaluation configural, metric, and scalar invariance are presented in Table 9. The multi-group configural model yielded satisfactory fit indices. Metric invariance was evaluated in the multi-group models next. It turned out that the deterioration of CFI and SRMR exceeds the recommended thresholds (Chen, 2007) that suggest the lack of metric measurement invariance. However, no local misspecifications were identified in applying the Saris et al. (2009) approach that suggests that the general result of metric noninvariance was caused by many small misspecifications rather than several large ones. Taking into account the large number of items (60) such results are not surprising.

Next, scalar invariance was evaluated in the multi-group model. Between the metric and scalar level, a deterioration of the indices was not exhibited (Chen, 2007). However, applying the local misspecification approach identified fewer than two invariant intercepts in some of the factors, which was not entirely supportive of scalar invariance.

Taken together, results suggest that the samples representing the different linguistic groups exhibited very good configural invariance of the Quick AIM scale after deleting 1 item. However metric and scalar measurement invariance is potentially problematic.

\section{DISCUSSION}

Obtaining empirical evidence to support a theory of the complexity of A-T theory has required a carefully planned, lengthy, iterative, and incremental development and testing process involving a variety of cross-sectional, longitudinal, and experimental designs (Bernard, 2013a). However, all previously published studies involved U.S. English-speaking samples, so the generalizability of Quick AIM scores and A-T theory remained an open question. The next logical step was to provide evidence of the measurement invariance of the model and the Quick AIM scales across samples of different languages and countries.

There was no reason to presume that all of the 60 Quick AIM items tapping the 15 motive dimensions in U.S. English samples should function well psychometrically in the different languages/cultures. Applying ESEM, the CFI suggested that the models were acceptable and the residual-based indices were more favorable for each of the five samples. 
However, there were mixed results in the multi-group invariance analyses, with support for configural but not metric and scalar invariance. Nevertheless, it was encouraging that 59 of the original 60 Quick AIM items performed well enough to test the 15-dimensional model proposed by A-T theory and the configural level. The fact that the 15 -dimensional model exhibited configural invariance across samples from four different languages is evidence of its utility for describing and assessing basic individual differences dimensions of human motivation. Due to the relatively small sample sizes for some of the groups, future research should cross-validate the results of the current study using a greater number of participants for each linguistic group in invariance analyses. Sample sizes of the groups might affect the interpretation of invariance analyses, as the RMSEA fit index is sensitive to sample sizes (Putnick \& Bornstein, 2016).

A new consideration arising from the present study is that the Illness Avoidance and Threat Avoidance scales possessed considerably lower internal consistency reliabilities across the samples than any other scales (Table 2). Cronbach's alpha is known to be sensitive to scale length (increasing as additional items with similar inter-item correlations are added). However, all of the Quick AIM scales, at four items, are brief and good values for Cronbach's alpha were obtained for the other 13 subscales. Although Threat Avoidance and Illness Avoidance have had adequate internal consistency reliabilities in lengthier versions of the scales in past studies, they have been consistently among the lowest (Bernard, 2013a). It may be that further investigation could yield better items to represent these two dimensions. However, it should be recognized that these two dimensions are characterized as "avoidance" and may be fundamentally different from the other dimensions that are primarily characterized as "approach" dimensions in A-T theory.

Important limitation of this study is that there were no non-Indo-European language families or non-Western countries represented in these samples. Five samples in this study share a similar historical and cultural frame that may differ from the other language families. Therefore, while they offer a test of the cross-linguistical applicability of the Quick AIM motives, samples may not be dissimilar enough to fully test the cross-linguistical applicability and factorial invariance of the A-T model. Still, these results are promising and they do suggest that this study should be replicated with more diverse linguistic and cultural samples. A difficulty that affects this type of research is that it is very time consuming and costly to conduct, requiring cross-national cooperation. Future research of A-T theory may focus on the importance of basic demographic variables, such as age, gender and cultural background. Some motive-traits (e.g., appearance, illness avoidance) may vary depending on such variables. For example, men place a premium on physical attractiveness in their choice of romantic partners. Accordingly, women tend to attract or retain mates by enhancing their physical attractiveness (e.g., Buss, 1988). This is reflected in the ways that women spend more time making themselves appear physically attractive compared with men (Aune \& Aune, 1994). Moreover, elderly people are considered to be more vulnerable to disease. Accordingly, illness avoidance behaviors are more frequent in elderly adults (Prohaska, Leventhal, Leventhal, \& Keller, 1985).

Given the rarity of an individual differences approach to the study of human motivation, this study suggests that there is promise in this method. If additional cross-cultural studies also produce evidence of these dimensions' relevance, AIM-Q scales may be useful adjuncts in the study of motivation. Brief, reliable, and valid scales based on A-T theory can be included in a variety of research studies and may add an additional dimension to clinical assessments. 


\section{REFERENCES}

Aune, R. K., \& Aune, K. S. (1994). The influence of culture, gender, and relational status on appearance management. Journal of Cross-Cultural Psychology, 25, 258-272. https://doi.org/10.1177/0022022194252006

Baumeister, R. F. (2016). Toward a general theory of motivation: Problems, challenges, opportunities, and the big picture. Motivation and Emotion, 40, 1-10. https://doi.org/10.1007/s11031-015-9521-y

Bernard, L. C. (2007). Assessing individual differences in motivation: Convergent validity of the Assessment of Individual Motives - Questionnaire [AIM-Q] and measures of aggression, cognition, playfulness, and sexuality. Individual Differences Research, 5, 158-174.

Bernard, L. C. (2009). Consensual and behavioral validity of a measure of adaptive individual differences dimensions in human motivation. Motivation and Emotion, 33, 303-319. https://doi.org/10.1007/s11031-009-9131-7

Bernard, L. C. (2010). Motivation and personality: Relationships between putative motive dimensions and the five factor model of personality. Psychological Reports, 106, 1-19. https://doi.org/10.2466/PR0.106.2.613-631

Bernard, L. C. (2012). Evolved individual differences in human motivation. In R. M. Ryan (Ed.), The Oxford Handbook of Human Motivation (pp. 381 407). Oxford, UK: Oxford University Press.

Bernard, L. C. (2013a). Manual for the Assessment of Individual Motives - Questionnaire. Department of Psychology, Loyola Marymount University, Los Angeles, CA.

Bernard, L. C. (2013b). Relationships between individual differences in motivation and borderline personality disorder, psychopathy, and maladjustment. Psychological Reports, 113, 129-161. https://doi.org/10.2466/14.02.09.PR0.113x14z9

Bernard, L. C., \& Hardy, D. J. (2014). Motivated behavioral outcomes affect ratings of attractiveness. Psychological Reports, 115, 849-871. https://doi.org/10.2466/21.02.PRO.115c30z0

Bernard, L. C., \& Lac, A. (2014a). Testing a multidimensional model of putative evolved human motives. Motivation and Emotion, 38, 47-64. https://doi.org/10.1007/s11031-013-9360-7

Bernard, L. C., \& Lac, A. (2014b). The incremental validity of motive "traits of action:" Predicting behavior longitudinally. Individual Differences Research, 12, 79-100.

Bernard, L. C., Lac, A., \& Lukaszewski, A. (2013, May). Motivation and personality: Relationships between QuickAIM motives and the HEXACO model of personality. Poster presented at the meeting of the Society for the Study of Motivation, Washington, DC.

Bernard, L. C., Mills, M., Swenson, L., \& Walsh, R. P. (2005). An evolutionary theory of human motivation. Genetic, Social, and General Psychology Monographs, 131, 129-184. https://doi.org/10.3200/MON0.131.2.129-184

Bernard, L. C., Mills, M., Swenson, L., \& Walsh, R. P. (2008). Measuring Motivation Multidimensionally: Development of the Assessment of Individual Motives-Questionnaire [AIM-Q]. Assessment, 15, 16-35. https://doi.org/10.1177/1073191107306131

Bugental, D. B. (2000). Acquisition of the algorithms of social life: A domain-based approach. Psychological Bulletin, 126, 187-219. https://doi.org/ 10.1037/0033-2909.126.2.187

Buss, D. M. (1988). The evolution of human intrasexual competition: Tactics of mate attraction. Journal of Personality and Social Psychology, 54 , 616-628. https://doi.org/10.1037/0022-3514.54.4.616

Byrne, B. M. (2012). Structural equation modeling with Mplus: Basic concepts, applications, and programming. New York, NY: Routledge/Taylor \& Francis. https://doi.org/10.4324/9780203807644

Byrne, B. M., Shavelson, R. J., \& Muthén, B. (1989). Testing for the equivalence of factor covariance and mean structures: The issue of partial measurement invariance. Psychological Bulletin, 105, 456-466. https://doi.org/10.1037/0033-2909.105.3.456

Campbell, D. T., \& Fiske, D. W. (1959). Convergent and discriminant validation by the multitrait-multimethod matrix. Psychological Bulletin, 56, 81-105. https://doi.org/10.1037/h0046016

Chen, F. F. (2007). Sensitivity of goodness of fit indexes to lack of measurement invariance. Structural Equation Modeling: A Multidisciplinary Journal, 14, 464-504. https://doi.org/10.1080/10705510701301834

Cieciuch, J., Davidov, E., Oberski, D. L., \& Algesheimer, R. (2015). Testing for measurement invariance by detecting local misspecification and an illustration across online and paper-and-pencil samples. European Political Science, 14, 521-538. https://doi.org/10.1057/eps.2015.64

Davidov, E., Meuleman, B., Cieciuch, J., Schmidt, P., \& Billiet, J. (2014). Measurement equivalence in cross-national research. Annual Review of Sociology, 40, 55-75. https://doi.org/10.1146/annurev-soc-071913-043137

Dawkins, R. (1982). The extended phenotype. Oxford, UK:W. H. Freeman.

Deci, E., \& Ryan, R. (1987). The support of autonomy and the control of behavior. Journal of Personality and Social Psychology, 53, 1024-1037. https://doi.org/10.1037/0022-3514.53.6.1024

Dunlap, K. (1919). Are there any instincts? Journal of Abnormal Psychology, 14, 35-50.

Hamilton, W. D. (1964). The genetical evolution of social behavior. Journal of Theoretical Biology, 7, 1-52. https://doi.org/10.1016/0022-5193(64)90038-4 Harriman, P. L. (1975). Handbook of psychological terms. Totowa, NJ: Littlefield, Adams.

Hogan, R. (1996). A socioanalytic perspective on the five-factor model. In J. S. Wiggins (Ed.), The five-factor model of personality: Theoretical perspectives (pp. 163-179). New York, NY: Guilford. 
Holt, E. B. (1931). Animal drive and the learning process: An essay toward radical empiricism. New York, NY: Holt.

Hoyle, R. H. (Ed.). (2012). Handbook of structural equation modeling. New York, NY: Guilford Press.

Hu, L. T., \& Bentler, P. M. (1998). Fit indices in covariance structure modeling: Sensitivity to underparameterized model misspecification. Psychological Methods, 3, 424-453. https://doi.org/10.1037//1082-989x.3.4.424

Hu, L. T., \& Bentler, P. M. (1999). Cutoff criteria for fit indexes in covariance structure analysis: Conventional criteria versus new alternatives. Structural Equation Modeling: A Multidisciplinary Journal, 6, 1-55. https://doi.org/10.1080/10705519909540118

International Test Commission. (2008). International Test Commission Guidelines for Translating and Adapting Tests. London, UK: The British Psychological Society. Retrieved from: www.InTestCom.org

James, W. (1890). Instinct. In Principles of Psychology (Vol. II, pp. 383-441). New York, NY: Holt.

Kenrick, D. T., Li, N. P., \& Butner, J. (2003). Dynamical evolutionary psychology: Individual decision rules and emergent social norms. Psychological Review, 110, 3-28. https://doi.org/10.1037/0033-295x.110.1.3

Lance, C. E., \& Vandenberg, R. J. (2002). Confirmatory factor analysis. In F. Drasgow \& N. Schmitt (Eds.), Measuring and analyzing behaviour in organizations: Advances in measurement and data analysis. San Francisco, SF: Jossey-Bass.

Lee, K., \& Ashton, M. C. (2004). Psychometric properties of the HEXACO Personality Inventory. Multivariate Behavioral Research, 39, 329-358. https://doi.org/10.1207/s15327906mbr3902_8

MacCallum, R. C., \& Austin, J. T. (2000). Applications of structural equation modeling in psychological research. Annual Review of Psychology, 51, 201-226. https://doi.org/10.1146/annurev.psych.51.1.201

MacCallum, R. C., Browne, M. W., \& Sugawara, H. M. (1996). Power analysis and determination of sample size for covariance structure modeling. Psychological Methods, 1, 130-149. https://doi.org/10.1037/1082-989X.1.2.130

Marsh, H.W., Ludtke, O., Muthén, B., Asparouhov, T., Morin, A. J. S., Trautwein, U., \& Nagengast, B. (2010). A new look at the big five factor structure through exploratory structural equation modeling. Psychological Assessment, 22, 471-491. https://doi.org/10.1037/a0019227

Marsh, H. W., Morin, A. J. S., Parker, P. D., \& Kaur, G. (2014). Exploratory structural equation modeling: An integration of the best features of exploratory and confirmatory factor analysis. Annual Review of Clinical Psychology, 10, 85-110. https://doi.org/10.1146/annurev-clinpsy-032813-153700

McClelland, D. C., Atkinson, J. W., Clark, R. W., \& Lowell, E. L. (1976). The achievement motive (2nd ed.). New York, NY: Irvington.

Morin, A. J., Boudrias, J. S., Marsh, H. W., Madore, I., \& Desrumaux, P. (2016). Further reflections on disentangling shape and level effects in person-centered analyses: An illustration exploring the dimensionality of psychological health. Structural Equation Modeling: A Multidisciplinary Journal, 23, 438-454. https://doi.org/10.1080/10705511.2015.1116077

Muthén, L. K., \& Muthén, B. 0. (1998-2012). Mplus user's guide (7th ed.). Los Angeles, CA: Authors.

Oettingen, G., Bulgarella, C., Henderson, M., \& Gollwitzer, P. M. (2004). Self-awareness, self-motives, and self-motivation. In R. A. Wright, J. Greenberg, \& S. S. Brehm (Eds.), Motivational analyses of social behavior: Building on Jack Brehm's contributions to psychology (pp. 225-244). Mahwah, NJ: Erlbaum.

Prohaska, T. R., Leventhal, E. A., Leventhal, H., \& Keller, M. L. (1985). Health practices and illness cognition in young, middle aged, and elderly adults. Journal of Gerontology, 40, 569-578. https://doi.org/10.1093/geronj/40.5.569

Putnick, D. L., \& Bornstein, M. H. (2016). Measurement invariance conventions and reporting: The state of the art and future directions for psychological research. Developmental Review, 41,71-90. https://doi.org/10.1016/j.dr.2016.06.004

Saris, W. E., Satorra, A., \& van der Veld, W. M. (2009). Testing structural equation models or detection of misspecifications? Structural Equation Modeling, 16, 561-582. https://doi.org/10.1080/10705510903203433

Tabachnick, B. G., \& Fidell, L. S. (2007). Using multivariate statistics (5th ed.). Boston, MA: Allyn \& Bacon/Pearson Education.

Tabachnick, B. G., \& Fidell, L. S. (2013). Using multivariate statistics (6th ed.). Upper Saddle River, NJ: Pearson Education.

Tolman, E. C. (1932). Purposive behaviour in animals and men. London, UK: Century/Random House.

Tooby, J., \& Cosmides, L. (1992). The psychological foundation of culture. In J. H. Barkow, L. Cosmides, \& J. Tooby (Eds.), The adapted mind (pp. 19136). New York, NY: Oxford University Press.

Vazsonyi, A. T., Ksinan, A., Mikuška, J., \& Jiskrova, G. (2015). The big five and adolescent adjustment: An empirical test across six cultures. Personality and Individual Differences, 83, 234-244. https://doi.org/10.1016/j.paid.2015.03.049

Warner, R. M. (2013). Applied statistics: From bivariate through multivariate techniques (2nd ed.). Thousand Oaks, CA: Sage. 


\section{APPENDIX \\ LANGUAGE VERSIONS OF THE QUICK AIM 60-ITEM SCALE}

KEY

Aggression: 1, 16, 31, 46

Threat Avoidance: 2, 17, 32, 47

Illness Avoidance: 3, 18, 33, 48

Environmental Inquisitiveness: 4, 19, 34, 49

Interpersonal Inquisitiveness: 5, 20, 35, 50

Sex: 6, 21, 36, 51

Appearance: 7, 22, 37, 52

Wealth: 8, 23, 38, 53

Mental: 9, 24, 39, 54

Physical: 10, 25, 40, 55

Commitment: 11, 26, 41, 56

Altruism: 12, 27, 42, 57

Social Exchange: 13, 28, 43, 58

Legacy: 14, 29, 44, 59

Meaning: 15, 30, 45, 60

\section{ENGLISH ITEMS}

1. I try to intimidate others to keep them from challenging me.

2. I spend a lot of effort to avoid physical threats of any kind.

3. I wash my hands before I eat anything, even a quick snack.

4. I make the effort to explore something I have not seen before.

5. I play with, jest, or mock people to discover their reactions.

6. I spend time thinking about how to have more sex.

7. I spend money and time on stylish hair cuts to improve my appearance.

8. I buy (or would buy) expensive or rare luxury goods so that others will know I am successful.

9. I show off my understanding of abstract or complex ideas so people will respect me.

10. I train hard to have as strong a body as I can.

11. I take time to offer emotional support and affection to the person I am involved with romantically.

12. I sacrifice (or would sacrifice) to help my distant relatives.

13. I take care of all my responsibilities to others.

14. I contribute to charitable organizations that do good work in the world.

15. I put a lot of effort into investigating different philosophies and approaches to life.

16. I use physical threats so that others know not to get in my way.

17. I stay clear of people who are engaged in a conflict situation for fear of harm.

18. I am very careful not to touch any surfaces after I wash my hands in a public place.

19. I am curious about new and different places because they may have something to offer.

20. I tease friends and acquaintances because it helps me understand them better.

21. I spend (or have spent) a lot of time and energy to have sex.

22. I spend money or time to improve my appearance such as getting a tan, spa treatments, or other similar things.

23. I like to have valuable things like cars, watches, or jewelry that other people notice. 
24. I engage in discussions or debates to develop a reputation as a smart and influential person.

25. I make the effort to stay in peak physical condition compared to others.

26. I make the effort to do the things that show I care for my spouse or partner.

27. I would let (or have let) distant relatives live with me temporarily if they needed shelter.

28. I do what is required to keep a contract or bargain I have made.

29. I donate my time, effort, or money to causes that promote health, reduce famine, or improve education in other countries.

30. I take time to investigate different religions and philosophies.

31.I use verbal threats so that others know not to get in my way.

32. I leave a situation when people become hostile toward each other.

33. I try to avoid any contact with people when there is an illness going around.

34. I explore unknown places to understand them better.

35. I like to play around with people to see how they react, even if they get angry at times.

36. I invest (or have invested) time seeking a lot of different sexual partners.

37. I buy expensive shoes or other accessories to look attractive.

38. I put a lot of time and energy into having expensive possessions.

39. I put a lot of effort into developing my own special skills and abilities to impress others.

40. I work on my body to be as physically competitive as I can.

41. I give of myself and my time in a romantic relationship.

42. I would take slight personal risks to my safety to help distant relatives.

43. I strive to be honest and fair.

44. I donate my time, effort, or money to non-profit community organizations that can make a better future for others.

45. I learn about various religions or philosophies to understand the purpose of life better.

46. I take physical risks so others will fear me.

47. I leave when a fight breaks out between people in order to stay safe.

48. I avoid traveling when infectious diseases are circulating.

49. I am interested in going to different places in order to learn something that could help me.

50.I try to understand others by playfully teasing them to see how they react.

51.I spend (or have spent) a lot of time pursuing sex.

52.I spend money on grooming (hair cuts, coloring, tanning) to look my best.

53. I spend a lot of money to buy things that make others envious.

54. I put effort into developing my talents and abilities in order to impress others.

55. I train hard to be to earn the reputation of being in peak physical condition.

56. I give what is necessary of my time and resources in romantic relationships.

57. I would invest time and effort to help a distant relative even if there were no immediate advantage to me.

58. I make an effort to treat others the way I want to be treated.

59. I contribute time, effort, and/or money to make the world better for future generations.

60. I study various philosophies of life and its meaning in order to find what is common in all.

\section{CROATIAN ITEMS}

1. Pokušavam zastrašiti druge kako bi ih spriječio da me izazivaju.

2. Dosta se trudim izbjeći fizičke opasnosti svih vrsta.

3. Perem ruke prije nego išta pojedem, makar i nešto na brzinu.

4. Trudim se istražiti mjesta koja do sada nisam vidio.

5. Poigravam se s ljudima, zadirkujem ih i podsmijavam im se kako bi vidio njihovu reakciju.

6. Provodim puno vremena razmišsjajući kako se više seksati.

7. Trošim novac i vrijeme na stilizirane frizure kako bi poboljšao izgled. 
8. Kupujem (ili bih kupovao) skupe i rijetke luksuzne stvari kako bi drugi znali da sam uspješan.

9. Razmećem se svojim razumijevanjem apstraktnih ili složenih ideja kako bi me ljudi poštivali.

10. Naporno treniram kako bih imao što snažnije tijelo.

11. Odvajam puno vremena kako bih pružio emocionalnu podršku i privrženost osobi s kojom se nalazim u romantičnoj vezi.

12. Žrtvujem se (ili bih se žrtvovao) kako bih pomogao svojoj daljoj rodbini.

13. Izvršavam sve svoje dužnosti prema drugima.

14. Doniram dobrotvornim organizacijama koje čine dobro u svijetu.

15. Ulažem puno truda u istraživanje različitih filozofija i pristupa životu.

16. Koristim se fizičkim prijetnjama kako bi drugi znali da mi ne trebaju stajati na putu.

17. Držim se podalje od ljudi uključenih u konfliktne situacije iz straha da ne bi nastradao.

18. Jako pazim da ne diram nikakve površine nakon što operem ruke na javnom mjestu.

19. Znatiželjan sam oko novih i drugačijih mjesta, jer bi mogla imati nešto za ponuditi.

20. Zadirkujem prijatelje i poznanike jer mi to pomaže da ih bolje upoznam.

21. Provodim (ili sam provodio/la) puno vremena i energije kako bih došao do seksa.

22. Trošim novac ili vrijeme kako bi poboljšao svoj izgled sunčanjem ili odlaskom na kvarcanje, spa tretmane i druge slične stvari.

23. Volim posjedovati vrijedne stvari poput automobila, satova ili nakita koje druge osobe mogu primjetiti.

24. Uključujem se u rasprave ili debate kako bih razvio reputaciju pametne i utjecajne osobe.

25. Trudim se da u odnosu na druge ostanem u naponu snage.

26. Trudim se raditi stvari koje mojem supružniku ili partneru pokazuju da mi je stalo do njega/nje.

27. Dopustio bih (ili sam dopustio) daljoj rodbini da privremeno žive kod mene ako im to zatreba.

28. Napraviti ću sve što je potrebno kako bih ispoštovao dogovor ili ugovor koji sam sklopio.

29. Doniram svoje vrijeme, trud ili novac događajima koji promoviraju zdravlje, smanjuju glad ili poboljšavaju obrazovanje u drugim zemljama.

30. Izdvajam vremena u istraživanje različitih religija i filozofija.

31. Koristim se verbalnim prijetnjama kako bi drugima dao/la do znanja da mi ne stoje na putu.

32. Napuštam situaciju u kojoj ljudi postanu agresivni (neprijateljski nastrojeni) jedni prema drugima.

33. Pokušavam izbjeći bilo koji kontakt s ljudima kada su bolesni.

34. Istražujem nepoznata mjesta kako bi ih bolje razumio.

35. Volim se poigravati s ljudima kako bih vidio kako će reagirati, čak iako se zbog toga znaju ponekad naljutiti.

36. Provodim (ili sam provodio) dosta vremena u traženju većeg broja različitih seksualnih partnera.

37. Kupujem skupe cipele i druge odjevne dodatke kako bih izgledao atraktivno.

38. Ulažem mnogo vremena i energije kako bih posjedovao skupocjene predmete.

39. Ulažem puno napora u razvijanje vlastitih posebnih vještina i sposobnosti kako bi impresionirao druge.

40. Radim na svom tijelu kako bi bio fizički spremniji u odnosu na ostale.

41. Dajem sebe i svoje vrijeme u romantičnoj vezi.

42. Malo bi riskirao svoju sigurnost kako bih pomogao svojoj daljoj rodbini.

43. Nastojim biti iskren i pošten.

44. Doniram svoje vrijeme, trud ili novac neprofitabilnim društvenim organizacijama koje mogu stvoriti bolju budućnost za druge.

45. Učim o različitim religijama ili filozofijama kako bih bolje razumio svrhu života.

46. Riskiram s fizičkim obračunima kako bi me se druge osobe bojale.

47. Otići ću kada izbije tuča između ljudi da bi ostao siguran/na.

48. Izbjegavam putovanja u vrijeme kada kruže zarazne bolesti.

49. Zainteresiran sam za odlazak na različita mjesta kako bi naučio nešto novo što bi mi moglo koristiti.

50. Pokušavam razumjeti druge na način da ih zadirkujem kako bi vidio kako će reagirati. 
51. Provodim puno vremena kako bi došao do seksa.

52. Trošim novac za dotjerivanje (frizura, bojanje kose, kvarcanje) kako bi izgledao najbolje što mogu.

53. Trošim mnogo novaca na kupnju stvari na kojima mi drugi zavide.

54. Ulažem trud u razvijanje svojih talenata i sposobnosti kako bih impresionirao druge.

55. Naporno treniram kako bih ostvario reputaciju nekoga tko je u stalnoj fizičkoj kondiciji.

56. Dajem, koliko god da je potrebno, svoje vrijeme i resurse u romantičnoj vezi.

57. Uložio bih vrijeme i trud pomagajući daljoj rodbini, čak iako od toga ne bih imao izravne koristi.

58. Trudim se ponašati prema drugima onako kako bih želio da se oni ponašaju prema meni.

59. Doprinosim svojim vremenom, trudom i/ili novcem kako bih učinio svijet boljim za nadolazeće generacije.

60. Proučavam različite filozofije života i njihovo značenje u svrhu traženja onoga što im je zajedničko.

\section{FRENCH ITEMS}

1. J'essaie d'intimider les autres pour les empêcher de me défier.

2. Je fais beaucoup d'effort pour éviter les menaces physiques en tous genres.

3. Je lave mes mains avant de manger quoi que ce soit, même pour un snack rapide.

4. Je fais l'effort d'explorer quelque chose que je n'ai pas vu avant.

5. Je joue avec, plaisante, me moque des gens pour découvrir leurs réactions.

6. Je passe du temps à penser à comment avoir plus de rapports sexuels.

7. Je dépense de l'argent et du temps dans des coupes de cheveux élégantes pour améliorer mon apparence.

8. J'achète (ou j'achèterais) des articles de luxe chers ou rares comme ça les autres sauront que je réussis.

9. J'affiche fièrement ma compréhension d'idées complexes ou abstraites comme ça les gens me respecteront.

10. Je m'entraîne dur pour avoir un corps aussi fort que je peux.

11. Je prends du temps pour offrir du support émotionnel et de l'affection à la personne avec qui je suis engagé amoureusement.

12.Je me sacrifie (ou me sacrifierais) pour aider mes parents éloignés.

13. Je prends soin de toutes mes responsabilités envers les autres.

14. Je contribue à des organisations caritatives qui font du bon travail dans le monde.

15. Je mets beaucoup d'effort pour étudier différentes philosophies et approches de la vie.

16. J'utilise des menaces physiques de manière à ce que les autres sachent qu'ils ne doivent pas se mettre sur mon chemin.

17. J'évite les gens qui sont engagés dans une situation de conflit par peur d'avoir mal.

18. Je fais très attention de ne pas toucher de surfaces après que j’ai lavé mes mains dans un espace public.

19. Je suis curieux à propos d'endroits nouveaux et différents parce qu'ils peuvent avoir quelque chose à offrir.

20. Je taquine mes amis et connaissances parce que cela maide à mieux les comprendre.

21. Je dépense (ou ai dépensé) beaucoup de temps et d'énergie pour avoir des rapports sexuels.

22. Je dépense de l'argent et du temps pour améliorer mon apparence comme en allant bronzer, en recevant des soins esthétiques ou d'autres choses similaires.

23. J'aime avoir des choses qui ont de la valeur comme des voitures, des montres ou des bijoux que les autres gens remarquent.

24. Je m’engage dans des discussions ou débats pour développer une réputation de personne intelligente et influente.

25. Je fais l'effort de rester au meilleur de ma condition physique comparé aux autres. 
26. Je fais l'effort de faire des choses qui montrent que je prends soin de mon épouse ou de ma partenaire.

27. Je laisserais (ou ai laissé) des proches vivre avec moi temporairement s'ils avaient besoin d'un abri.

28. Je fais ce qui est exigé pour honorer un contrat ou marché que j'ai fait.

29. Je donne mon temps, des efforts, ou de l'argent à des causes qui promeuvent la santé, réduisent la famine ou améliorent l'éducation dans d'autres pays.

30. Je prends du temps pour étudier différentes religions et philosophies.

31. J'utilise des menaces verbales de manière à ce que les autres sachent qu'ils ne doivent pas se mettre sur mon chemin.

32. Je quitte une situation lorsque les gens deviennent hostiles les uns envers les autres.

33. J'essaie d'éviter tout contact avec des gens lorsqu'il y a une maladie qui tourne.

34. J'explore des endroits inconnus pour mieux les comprendre.

35. J'aime m'amuser avec les gens pour voir comment ils réagissent, même s'ils se mettent parfois en colère.

36. J'investis (ou ai investi) du temps à chercher beaucoup de partenaires sexuels différents.

37. J'achète des chaussures chères ou d'autres accessoires pour avoir l'air attirant(e).

38. Je mets beaucoup de temps et d'énergie pour avoir des biens chers.

39. Je fais beaucoup d'effort pour développer mes propres compétences et capacités spéciales pour impressionner les autres.

40. Je travaille sur mon corps pour être le plus physiquement compétitif que je peux.

41. Je donne de moi-même et de mon temps dans une relation amoureuse.

42. Je prendrais de légers risques personnels pour ma sécurité pour aider des parents éloignés.

43. Je m'efforce d'être honnête et juste.

44. Je donne mon temps, des efforts, ou de l'argent à des organisations communautaires sans but lucratif qui peuvent contribuer à un meilleur futur pour les autres.

45. J'apprends sur diverses religions ou philosophies pour mieux comprendre le but de notre vie.

46. Je prends des risques physiques comme ça les autres me craindront.

47. Je pars lorsqu'une bagarre éclate entre des gens pour rester en sécurité.

48. J'évite de voyager lorsque des maladies infectieuses circulent.

49. Je suis intéressé d'aller dans des endroits différents pour apprendre quelque chose qui pourrait m'aider.

50. J'essaie de comprendre les autres en les taquinant malicieusement pour voir comment ils réagissent.

51. Je passe (ou ai passé) beaucoup de temps à chercher à avoir des rapport sexuels.

52. Je dépense de l'argent en tenues (coupes de cheveux, couleurs, bronzage) pour apparaitre sous mon meilleur jour.

53. Je dépense beaucoup d'argent pour acheter des choses qui rendent les autres envieux.

54. Je fais des efforts pour développer mes talents et capacités pour impressionner les autres.

55. Je mentraine dur pour gagner la réputation d'être au meilleur de ma condition physique.

56. Je donne ce qui est nécessaire de mon temps et de mes ressources dans des relations amoureuses.

57. J'investirais du temps et de l'effort pour aider un parent éloigné même s'il n'y avait pas d'avantage immédiat pour moi.

58. Je fais un effort pour traiter les autres de la manière avec laquelle je veux être traité.

59. Je contribue avec mon temps, mes efforts et/ou mon argent pour rendre le monde meilleur pour les générations futures.

60. J'étudie diverses philosophies de vie et leur sens pour trouver ce qui est commun à toutes.

\section{GERMAN ITEMS}

1. Ich versuche Andere einzuschüchtern, damit sie mich nicht herausfordern.

2. Ich verwende viel Anstrengung darauf, körperliche Bedrohungen jeglicher Art zu vermeiden. 
3. Ich wasche meine Hände bevor ich etwas esse, auch wenn es nur ein schneller Snack ist.

4. Ich bemühe mich etwas zu entdecken, was ich noch nie zuvor gesehen habe.

5. Ich spiele mit anderen Menschen, mache Scherze über sie oder veralbere sie, um ihre Reaktionen zu beobachten.

6. Ich denke häufig darüber nach, wie ich mehr Sex haben kann.

7. Ich investiere Zeit und Geld in stylische Frisuren, um mein Aussehen zu verbessern.

8. Ich kaufe teure und seltene Luxusgüter, damit andere glauben, dass ich erfolgreich bin / Ich würde teure und seltene Luxusgüter kaufen, damit andere glauben, dass ich erfolgreich bin.

9. Ich zeige, dass ich abstrakte oder komplexe Ideen verstehe, damit mich andere Menschen respektieren.

10. Ich trainiere hart, um einen möglichst starken Körper zu bekommen.

11. Ich nehme mir die Zeit, der Person mit der ich eine romantische Beziehung habe, emotionale Unterstützung und Zuneigung zu geben.

12. Ich gehe Opfer ein, um entfernten Verwandten zu helfen / Ich würde Opfer eingehen, um entfernten Verwandten zu helfen.

13. Ich nehme meine Verantwortungen gegenüber Anderen war.

14. Ich trage zu mildtätigen Organisationen bei, die in der Welt Gutes tun.

15. Ich verwende viel Anstrengung darauf, verschiedene Lebensphilosophien und Lebenseinstellungen kennenzulernen.

16. Ich setze körperliche Drohungen ein, damit Andere mir nicht in die Quere kommen.

17. Ich meide Personen, die sich in konfliktreichen Situationen befinden, da ich Angst habe verletzt zu werden.

18. Ich bemühe mich keine Oberflächen anzufassen, nachdem ich meine Hände in einem öffentlich zugänglichen Raum gewaschen habe.

19. Ich bin neugierig auf neue und unterschiedliche Orte, da sie möglicherweise etwas zubieten haben.

20. Ich necke Freunde und Bekannte, da es mir hilft, sie besser zu verstehen.

21. Ich verwende viel Zeit und Energie darauf Sex zu haben / Ich habe viel Zeit und Energie darauf verwandt Sex zu haben.

22. Ich verwende Geld und Zeit, um mein Aussehen zu verbessern (beispielsweise für Solariumbesuche, Schönheitsbehandlungen oder ähnlichen).

23. Ich besitze gerne wertvolle Dinge wie Autos, Uhren oder Schmuck, die andere Leute wahrnehmen.

24. Ich engagiere mich in Diskussionen oder Debatten, um einen Ruf als smarte und einflussreiche Person zu entwickeln.

25. Ich bemühe mich im Vergleich zu Anderen in bester körperlicher Verfassung zu bleiben.

26. Ich strenge mich an Dinge zu tun, die zeigen, dass ich mich um meinen Ehepartner oder Partner kümmere.

27. Ich habe entfernte Verwandte bei mir vorübergehend wohnen lassen, wenn sie eine Unterkunft brauchten / Ich würde entfernte Verwandte bei mir vorübergehend wohnen lassen, wenn sie eine Unterkunft brauchen.

28. Ich tue was nötig ist, um einen Vertrag oder ein Geschäft, das ich abgeschlossen habe, einzuhalten.

29. Ich verwende meine Zeit, Anstrengung oder Geld für Zwecke, die in anderen Ländern Gesundheit fördern, Hunger reduzieren oder die Bildung verbessern.

30. Ich nehme mir die Zeit verschiedene Religionen und Philosophien kennenzulernen.

31. Ich setze verbale Drohungen ein, damit Andere mir nicht in die Quere kommen.

32. Ich verlasse Situationen, in denen Menschen feindselig zu einander werden.

33. Ich vermeide jeglichen Kontakt mit Menschen, wenn eine Krankheit umgeht.

34. Ich erkunde unbekannte Orte genau, um sie besser zu verstehen.

35. Ich spiele gerne mit anderen Menschen, um zu sehen wie sie reagieren, auch wenn sie hin und wieder ärgerlich werden. 
36. Ich verwende/verwendete viel Zeit darauf viele unterschiedliche Sexualpartner zu haben.

37. Ich kaufe teure Schuhe oder andere Accessoires um attraktiv auszusehen.

38. Ich investiere eine Menge Zeit und Energie darauf teure Güter zu besitzen.

39. Ich strenge mich an, meine eigenen speziellen Fähigkeiten und Fertigkeiten zu entwickeln, um damit Andere zu beeindrucken.

40. Ich arbeite an meinem Körper, um physisch so konkurrenzfähig wie möglich zu sein.

41. Ich bringe mich selbst und meine Zeit in eine romantische Beziehung ein.

42. Ich würde ein kleines persönliches Sicherheitsrisiko eingehen, um entfernten Verwandten zu helfen.

43. Ich bemühe mich ehrlich und fair zu sein.

44. Ich spende gemeinnützigen Organisationen, die eine bessere Zukunft für Andere schaffen können, meine Zeit, Anstrengung oder Geld.

45. Ich informiere mich über verschiedene Religionen oder Philosophien, um den Sinn des Lebens besser zu verstehen.

46. Ich gehe körperliche Risiken ein, damit Andere mich fürchten.

47. Ich verlasse Situationen, wenn es zwischen Personen zu Kämpfen kommt, damit mir nichts passiert.

48. Ich vermeide Reisen, wenn ansteckende Krankheiten zirkulieren.

49. Ich bin daran interessiert unterschiedliche Orte zu besuchen, um etwas zu lernen was mir helfen könnte.

50. Ich versuche andere zu verstehen, indem ich sie spasseshalber aufziehe, um zu sehen, wie sie reagieren.

51. Ich verwende/verwendete viel Zeit darauf, Sex zu haben.

52. Ich verwende Geld darauf mich zurechtzumachen (Haarschnitt, Haare färben, braun zu werden) um bestmöglich auszusehen.

53. Ich gebe viel Geld aus, um Dinge zu kaufen, die andere eifersüchtig machen.

54. Ich strenge mich an, meine Talente und Fähigkeiten zu entwickeln, um Andere zu beeindrucken.

55. Ich trainiere hart, um den Ruf zu erlangen, in einem physischen Topzustand zu sein.

56. Ich investiere in romantischen Beziehungen die Zeit und Ressourcen, die nötig sind.

57. Ich würde Zeit und Anstrengung investieren, um einem entfernten Verwandten zu helfen, auch wenn mir dabei kein unmittelbarer Vorteil entstehen würde.

58. Ich bemühe mich andere so zu behandeln, wie ich selbst gerne behandelt werden möchte.

59. Ich verwende Zeit, Anstrengung und/oder Geld darauf, die Welt für zukünftige Generationen zu verbessern.

60. Ich studiere verschiedene Lebensphilosophien und ihre Bedeutung um herauszufinden, was ihnen allen gemeinsam ist.

\section{POLISH ITEMS}

1. Staram się zastraszyć innych, aby nie rywalizowali ze mną.

2. Wkładam wiele wysiłku, aby uniknąć jakichkolwiek fizycznych zagrożeń.

3. Przed każdym jedzeniem myję ręce, nawet jeśli jest to szybka przekąska.

4. Staram się dokładnie badać rzeczy, które widzę po raz pierwszy.

5. Gram z ludźmi, kpię z nich lub drwię, aby zobaczyć, jaka będzie ich reakcja.

6. Dużo się zastanawiam, co zrobić, aby częściej uprawiać seks.

7. Poświęcam pieniądze i czas na dobrego fryzjera, żeby poprawić swój wygląd.

8. Kupuję (lub kupował(a)bym) drogie lub rzadkie przedmioty luksusowe, żeby inni widzieli, że jestem człowiekiem sukcesu.

9. Pokazuję innym, że rozumiem jakieś abstrakcyjne lub złożone problemy, aby mnie szanowali.

10. Ciężko trenuję, aby mieć jak najsilniejsze ciało. 
11. Poświęcam czas, aby dać emocjonalne wsparcie i czułość osobie, którą kocham.

12. Poświęcam się (lub poświęcił(a)bym) się, aby pomóc swoim dalszym krewnym.

13. Wypełniam wszystkie swoje zobowiązania wobec innych.

14. Daję datki na organizacje charytatywne, które czynią coś dobrego dla świata.

15. Wkładam wiele wysiłku w badanie różnych filozofii i podejść do życia.

16. Stosuję groźby fizyczne, aby inni nie wchodzili mi w drogę.

17. Z obawy przed niebezpieczeństwem trzymam się z dala od ludzi zaangażowanych w jakiekolwiek konflikty.

18. Bardzo uważam, aby po umyciu rąk nie dotykać żadnych powierzchni w miejscu publicznym.

19. Ciekawią mnie nowe, inne miejsca, bo mogą oferować coś szczególnego.

20. Dokuczam przyjaciołom i znajomym, ponieważ pozwala mi to lepiej ich rozumieć.

21. Poświęcam wiele czasu i energii na uprawianie seksu.

22. Poświęcam czas i pieniądze, aby poprawić swój wygląd, np. chodzę do solarium, spa itd.

23. Lubię mieć cenne rzeczy, na które inni zwracają uwagę, takie jak: samochody, zegarki lub biżuterię.

24. Angażuję się w dyskusje lub debaty, aby wyrobić sobie reputację osoby mądrej i wpływowej.

25. Staram się, aby w porównaniu $z$ innymi mieć bardzo dobrą kondycję fizyczną.

26. Staram się robić rzeczy świadczące o tym, że troszczę się o swego współmałżonka lub partnera/ partnerkę.

27. Pozwalam (lub pozwolił(a)bym) swoim dalszym krewnym mieszkać ze mną przez jakiś określony czas, jeśli potrzebowaliby dachu nad głową.

28. Robię wszystko, co jest wymagane w umowie, na którą się godzę.

29. Przeznaczam swój czas, wysiłek lub pieniądze na organizacje, które promują zdrowie, zmniejszają klęskę głodu lub wspierają edukację w innych krajach.

30. Poświęcam swój czas na zgłębianie różnych religii i filozofii.

31. Stosuję groźby werbalne, aby inni nie wchodzili mi w drogę.

32. Kiedy w jakiejś sytuacji ludzie zaczynają być wobec siebie wrodzy, odchodzę stamtąd.

33. Kiedy panują jakieś choroby, staram się unikać kontaktu z innymi ludźmi.

34. Badam miejsca, których nie znam, bo chcę je zrozumieć.

35. Lubię igrać z ludźmi, aby zobaczyć ich reakcje, nawet jeśli się wtedy czasem złoszczą.

36. Poświęcam czas na poszukiwanie wielu różnych partnerów seksualnych.

37. Kupuję drogie buty lub inne dodatki, aby wyglądać atrakcyjnie.

38. Inwestuję wiele czasu i energii, żeby posiadać kosztowne rzeczy.

39. Wkładam wiele wysiłku w rozwijanie swoich zdolności i umiejętności, aby zrobić wrażenie na innych.

40. Pracuję nad swoim ciałem, aby było fizycznie bezkonkurencyjne.

41. Daję siebie i swój czas osobie, którą kocham.

42. Zaryzykował(a)bym nieco własne bezpieczeństwo, aby pomóc dalekiemu krewnemu.

43. Staram się być uczciwy/a i sprawiedliwy/a.

44. Przeznaczam swój czas, wysiłek lub pieniądze dla społecznych organizacji non-profit, które dbają o lepszą przyszłość dla innych.

45. Zdobywam informacje o różnych religiach lub filozofiach, żeby lepiej rozumieć cel życia.

46. Podejmuję fizyczne ryzyko, aby inni bali się mnie.

47. Gdy między ludźmi rozpoczyna się bójka, oddalam się, aby pozostać bezpiecznym.

48. Unikam podróży, gdy jest dużo zachorowań na choroby zakaźne.

49. Z zainteresowaniem chodzę w różne miejsca, żeby nauczyć się czegoś, co może mi się przydać.

50. Staram się zrozumieć innych, drocząc się z nimi i obserwując ich reakcję.

51. Poświęcam wiele czasu na poszukiwanie seksu.

52. Wydaję pieniądze na różne zabiegi, żeby wyglądać jak najlepiej (nowe fryzury, farbowanie włosów, opalanie).

53. Wydaję dużo pieniędzy na rzeczy, których inni będą mi zazdrościć.

54. Staram się rozwijać moje talenty i zdolności, aby zrobić wrażenie na innych. 
55. Ciężko ćwiczę, aby mieć opinię osoby w doskonałej formie fizycznej.

56. W moim związku z kochaną osobą poświęcam konieczny czas i środki.

57. Poświęcił(a)bym czas i wysiłek, aby pomóc dalekiemu krewnemu, nawet jeśli nie miał(a)bym $\mathrm{z}$ tego bezpośrednich korzyści.

58. Staram się traktować ludzi tak, jak sam/a chcę być traktowany/a.

59. Poświęcam swój czas, wysiłek i/lub pieniądze, aby sprawić, że świat będzie lepszy dla przyszłych pokoleń.

60. Zdobywam wiedzę na temat różnych filozofii życia i jego sensu, aby znaleźć to, co je łączy. 